STUDI

FRANCESI

\section{Studi Francesi}

Rivista quadrimestrale fondata da Franco Simone

194 (LXV | II) | 2021

Baudelaire et son cénacle

\title{
De la pensée de l'Histoire au jeu littéraire. Études médiévales en l'honneur de Dominique Boutet
}

\section{Paola Cifarelli}

\section{(2) OpenEdition}

1 Journals

\section{Édition électronique}

URL : https://journals.openedition.org/studifrancesi/44575

DOI : 10.4000/studifrancesi.44575

ISSN : 2421-5856

Éditeur

Rosenberg \& Sellier

\section{Édition imprimée}

Date de publication : 1 août 2021

Pagination : 339-342

ISSN : 0039-2944

\section{Référence électronique}

Paola Cifarelli, «De la pensée de l'Histoire au jeu littéraire. Études médiévales en l'honneur de Dominique Boutet », Studi Francesi [En ligne], 194 (LXV | II) | 2021, mis en ligne le 06 septembre 2021, consulté le 14 octobre 2022. URL : http://journals.openedition.org/studifrancesi/44575 ; DOI : https://doi.org/ 10.4000/studifrancesi.44575

Ce document a été généré automatiquement le 14 octobre 2022.

\section{(c)}

Creative Commons - Attribution - Pas d'Utilisation Commerciale - Pas de Modification 4.0 International - CC BY-NC-ND 4.0

https://creativecommons.org/licenses/by-nc-nd/4.0/ 


\title{
De la pensée de l'Histoire au jeu littéraire. Études médiévales en l'honneur de Dominique Boutet
}

\author{
Paola Cifarelli
}

\section{RÉFÉRENCE}

De la pensée de l'Histoire au jeu littéraire. Études médiévales en l'honneur de Dominique Boutet. Études réunies par S. DUCHET, M.-P. HALARY, S. LEFÈVRE, P. MORAN et J.-R. VALETTE, Paris, Honoré Champion, 2019, «Nouvelle Bibliothèque du Moyen Âge» 127, 922 pp.

1 Cet imposant recueil de mélanges dédiés à Dominique Boutet montre l'importance que les études critiques du dédicataire ont eue dans un grand nombre de domaines de la littérature médiévale. Les articles sont organisés en quatre parties, consacrées respectivement aux «Rois imaginaires: Charlemagne, Arthur, Alexandre, Noble et les autres», au genre épique («La Chanson de geste: formes et significations»), à la réflexion sur les formes («Formes littéraires, conscience historique et conscience de soi») et à l'entrecroisement de genres différents («Poétiques de l'entre-deux et interférences génériques»). Le nombre des articles réunis dans ce volume ne permettra que de rendre compte sous une forme très succincte du contenu de chacun d'entre eux.

Dans la première partie, trois contributions sont consacrées à l'image royale des romans arthuriens dans ses nombreuses facettes. Laurence MATHEY-MAILLE (Arthur pensif au couteau: l'image du roi Arthur dans l'“Âtre périlleux", pp. 55-63) analyse l'image d'Arthur, et particulièrement celle du couteau qui se brise, dans l'Âtre périlleux, qui met en question les valeurs d'harmonie et d'idéal traditionnellement associées à la cour de la Table Ronde, tandis que Catherine NICOLAS ("Certes, voirement est il voirs». La vraie image $d u$ roi Arthur dans les manuscrits cycliques du "Lancelot-Graal" (fin du XIII - début du XIV siècle), pp.64-75) étudie les images figurant le roi dans les cinq manuscrits les plus anciens du Lancelot-Graal, et plus particulièrement celle où il est attaqué pendant son 
voyage vers la Douloureuse Garde. Une analyse plus transversale est menée dans les autres articles de cette section; les représentations de l'anniversaire du couronnement sont l'objet de la contribution de Sophie ALBERT, qui prend en compte quatre mises en prose: Lancelot, Tristan, la Suite Merlin post-vulgate et le Roman de Guiron (L'anniversaire $d u$ couronnement royal dans quelques romans arthuriens en prose, pp. 76-86). La vision critique de la royauté à travers le portrait de rois et reines faibles et pleins de vices brossé par l'auteur de Sone de Nansay et l'éloge du monarque qui accède au trône pour ses seuls mérites font l'objet de l'article de Claude LACHET (Images contrastées de la royauté dans "Sone de Nansay", pp. 87-98), tandis qu'à travers l'étude du portrait de Charlemagne dans les trois ouvrages qui renferment l'histoire des Neuf Preux, à savoir le traité de Sébastien Mamerot, l'Istoire des neuf preux du ms. Paris, BnF, fr. 12598 et le Triomphe des Neuf Preux imprimé, Anne salamon essaie de dégager le type de savoir convoqué dans ces compilations (Charlemagne: textes et images dans les compilations sur les Neuf Preux, pp. 99-110). L'attention se focalise ensuite sur le portrait royal dans les genres autres que la chanson de geste; le portrait d'Alexandre occupe Sandrine HÉRICHÉ-PRADEAU (L"Alexandre" de Vasque de Lucène: l'histoire à la lisière du mythe, pp.111-123), qui se penche sur la traduction française inédite de l'Historia Alexandri de Quinte Curce pour montrer la vocation d'historien qui anime le traducteur, par-delà les liens qui l'unissent à la tradition mythique véhiculée par les écrivains classiques et chrétiens. Roger BELLoN analyse l'image du monarque animalier du Roman de Renart et le portrait positif qu'en fournit l'auteur de la branche XIV («Plus sinple que uns colons»: un portrait inédit du roi Noble dans la branche intitulée "Renart le Noir", pp. 124-136); la colère du roi Richard I ${ }^{\mathrm{er}}$ Cœur de Lion dans la chronique de la troisième croisade et les contradictions apparentes que l'émotion princière véhicule sont étudiées par Marion BONANSEA (Expression des émotions et écriture de l'histoire. Le courroux de Richard I ${ }^{\text {er }}$ dans l'"Estoire de la guerre sainte", pp. 137-147). Catherine CROIZY-NAQUET interroge les Gesta Philippi Augusti, biographie rédigée du vivant du roi par le médecin Rigord, et étudie l'hybridité de son écriture de l'histoire, particulièrement empreinte d'esprit apologétique (Rigord, Philippe Auguste et la croisade, pp. 148-160); cette nécessité d'affirmer l'autorité royale dans les moments de crise anime également la Philippide, qui inscrit le portrait de Philippe Auguste dans la dimension de l'épopée au lendemain de la bataille de Bouvines (MarieMadeleine CASTELLANI, Chanter la gloire de Philippe Auguste dans la "Philippide" de Guillaume le Breton, pp.161-169). L'exemplum du Barlaam et Josaphat consacré au roi triste entendant sonner la trompette de la mort est étudié par Fabio zinelLI dans les réélaborations fournies par Wautriquet de Couvin dans le Dit des quatre gleves (ms. Paris, BnF, fr. 1708) et par Jean de Condé dans le Dit du roi et des hiermittes (Les usages d'un "miroir aux princes": un exemplum du "Barlaam et Josaphat" entre Gui de Cambrai, Wautriquet de Couvin et Jean de Condé, pp. 170-190). Des problèmes de datation du poème d'Adam de la Halle consacré à Charles d'Anjou, roi de Sicile à l'époque des vêpres siciliennes, sont au cœur de l'article de Michelle szKILNIK (Le "Livre des bons faits du roi de Sicile": Adam de la Halle biographe de Charles d'Anjou, pp. 191-200), tandis que Philippe MÉNARD (Guerre entre Mongols: l'empereur Khoubilai Khan contre le prince Nayan dans le texte de Marco Polo, essai de critique textuelle, pp. 201-210) soumet un épisode du Devisement du monde de Marco Polo à une comparaison entre les différentes versions de l'ouvrage et avec deux autres textes, un en persan et un autre en chinois dont les traductions sont fournies en annexe, pour prouver que le voyageur fut effectivement un témoin oculaire. La version de l'épisode connu comme Justice de l'empereur Othon fournie par le seul témoin français du Tombel de Chartrose (1334-1339) est soumise à une analyse destinée à en mettre en 
évidence l'originalité dans l'article de Pierre-Yves BADEL (Le jugement de l'empereur. À propos du "Tombel de Chartreuse" (récit 12): comment l'empereur Othon fit occire un comte injustement, pp. 211-224). Catherine GAULLIER-BOUgasSAS (Jean de Courcy et son idéal de la royauté dans la "Bouquechardière": la Vierge à l'enfant, Nectanabus et le dieu Amon, pp. 205-234) étudie l'image d'Alexandre dans les descriptions des deux séjours de celuici en Égypte figurant dans la Bouquechardière, afin de montrer que le texte véhicule une réinterprétation des faits à la lumière des idéaux de Jean de Courcy sur la royauté.

La deuxième section s'ouvre sur l'article de Jean-Pierre MARTIN (Le Jongleur dans ses œuvres. Portrait en creux d'une fiction énonciative, pp. 237-248), consacré aux interventions de l'interprète dans les chansons de geste, où celui-ci prête sa voix à l'auteur, au narrateur et au jongleur implicite pour fournir aux lecteurs une représentation littéraire de la performance orale. Patrick MORAN (Comment s'appelle la "Chanson de Roland"? Du titre au genre, pp. 249-260) analyse les désignations du texte dans les différents témoins qui nous ont transmis la Chanson de Roland, pour montrer que la distinction roman-chanson de geste résulte plus des perspectives modernes que des frontières établies par les lecteurs médiévaux. Philippe HAUGEARD (Ressorts et enjeux de la procédure judiciaire: le procès de Ganelon dans les versions d'Oxford et de Châteauroux de la "Chanson de Roland", pp. 261-278) revient sur l'épisode bien connu du procès de Ganelon dans la Chanson de Roland pour prouver que le témoignage du texte de Châteauroux mérite d'être réévalué en raison du choix d'une stratégie narrative indépendante et cohérente. La fascination éprouvée par Jorge Luis Borges pour l'anecdote du jongleur Taillefer chantant la Chanson de Roland à la bataille de Hastings fait l'objet de l'article de Hubert HECKMANN (La performance du jongleur normand à Hastings: le rôle d'une anecdote dans la réception créatrice de la "Chanson de Roland", pp. 279-285), tandis que Jean MAURICE étudie la manière dont le célèbre manuel d'histoire de la littérature française de Lagarde et Michard présente la Chanson de Roland (La "Chanson de Roland" dans le "Lagarde et Michard", pp. 286-294). Les concepts d'innovation et d'erreur, d'original et de meilleur témoin sont discutés par Edward A. HEINEMANN à partir d'une analyse stylistique des vers 37-38 du Charroi de Nîmes et des réélaborations figurant dans les versions parvenues jusqu'à nous (Les rythmes et la transmssion de deux vers du (des?) "Charroi de Nîmes", pp. 295-304). C'est encore la pratique du remaniement qui fait l'objet de l'étude de l'épisode de la mort de Maugis, cousin de Renaut de Montauban, dans la chanson de geste homonyme et dans les remaniements des XIV et $\mathrm{Xv}^{\mathrm{e}}$ siècles (Sarah BAUDELLE-MiChels, Les morts de "Maugis d'Aigremont", pp. 305-314); Muriel отT se penche sur les numéraux cardinaux utilisant un système de multiplication par dix qui figurent dans la Chevalerie Ogier (Quatre-dix, sept-dix, douze-dix: une autre expression du système de numération décimale?, pp.315-320), tandis que Jean-Marie ARDOUIN analyse le rapport symbolique qui unit le héros épique à certains lieux privilégiés à partir de l'éparpillement spatial caractérisant la chanson d'Aiol ("Aiol": le héros et les lieux, un parcours révélateur et individualisant?, pp. 321-332). La question de la frontière entre épopée et roman est étudiée par François SUARD à partir du témoignage d'Othevien, texte qui mêle les traits de la chanson de geste avec des motifs typiques du roman et de la chanson d'aventures (Les habits surprenants de la chanson de geste. À propos d"'Othevien" (ms Oxford, Bodleian Library, Hatton 100), pp. 333-348). L'identification de quelques-unes des sources utilisées par le compilateur du Huon en prose pour la reformulation des péripéties du héros en contexte bourguignon est au centre de l'article de Caroline CAZANAVE (Quand "Huon de Bordeaux" modifie son voyage en passant par la cour de Philippe le 
Bon (À propos de quelques trafics intertextuels repérés dans la prose), pp. 349-359), tandis que l'analyse de quelques références intertextuelles utiles pour mieux cerner la période et le milieu de création de la chanson de Florence de Rome menée par Emmanuelle POULAINgaUtRet (Mobilis in mobili. "Florence de Rome" et les épopées dites 'tardives', pp. 360-369) montre l'importance des échos d'autres textes pour l'étude des chansons tardives. Celles-ci font encore l'objet des trois études suivantes, consacrées respectivement à Lion de Bourges et plus particulièrement au brouillage d'identités et de valeurs caractérisant l'arrière-fond de cette chanson (Claude Roussel, Le monde incertain de "Lion de Bourges", pp. 370-379); à Galien le Restoré en prose, avec une analyse de l'affrontement entre couples de valeurs antithétiques dans l'épisode où le héros part au secours de sa mère (Bernard GUIDOT, "Galien le Restoré" en prose: élan héroïque, âmes noires et vertu outragée, pp. 380-389) et à la prose d'Anseïs de Gascogne, étudiée dans ses particularités lexicales par Jean-Charles HERBIN (Traits du lexique de la prose d'“Anseïs de Gascogne" copiée par David Aubert, pp. 390-400). Les articles d'Antonella NEGRI (Les "Aspramonte" en vers aux XVe et $\mathrm{XVI}^{\mathrm{e}}$ siècles, pp. 401-408), Jean-Baptiste CAMPs (Des lectrices de chansons de geste aux XIII-XVe siècles, pp. 409-426) et Joël H. GRISWARD (Signes et insignes du pouvoir dans quelques textes épiques de l'Afrique de l'Ouest et du monde indo-européen, pp. 427-438) achèvent cette partie.

4 La troisième section réunit seize articles qui interrogent des œuvres mettant en question la frontière des genres telle que la critique moderne la définit, ou portant un témoignage sur la réalité contemporaine. En prenant comme point de départ l'analyse de l'un des Miracles de saint Benoît en latin, Dominique BARTHÉLEMY (Entendre une épopée et s'enfuir en tremblant (Raoul Tortaire, "Miracles de saint Benoit", VIII.36), pp. 441-447) s'attarde sur des textes écrits en laisses n'appartenant pas au genre des chansons de geste, tandis que des chansons de geste empruntant des techniques de la chronique ou du roman sont étudiées par Marianne AILEs ( $A u$ carrefour des genres: chansons de geste historiques ou chroniques sous la forme de la chanson de geste, pp. 448-458). Blandine LONGHI soumet Raoul de Cambrai à une lecture anthropologique qui met en évidence l'importance d'une réflexion sur l'essence de la violence (L'impossible sortie de la violence? Une lecture girardienne de "Raoul de Cambrai", pp.458-467), et Jean-Claude VALLECALLE aborde la tradition épique en franco-italien (L'inscription du passé dans "Huon d'Auvergne", pp. 468-477). Le poème consacré au saint-roi anglo-saxon Edouard composé par Matthieu Paris entre 1236 et 1239 mêle, quant à lui, le récit historiographique et le mode d'écriture hagiographique (Françoise LAURENT, «Li Normant la victorie unt, e li Engleis descunfit sunt». La conquête normande et la "Estoire de Seint Aedward le rei" de Matthieu Paris, pp.478-488), tandis qu'une chronique versifiée de la conquête de l'Irlande par les colons venant du Pays de Galles dans la seconde moitié du XII siècle rédigée en langue vulgaire occupe Keith BUSBY, qui propose une lecture valorisante de ce texte où le problème du genre auquel il appartiendrait est particulièrement épineux (Pour une lecture de la "Geste des Engleis en Yrlande", pp. 489-500). Jean-René valetTE (Idéal et idéel: entre littérature et histoire, la question du merveilleux, pp. 501-513) prend en compte le concept de merveilleux et les problèmes de méthode que celui-ci soulève quant au statut du texte littéraire médiéval, entre approche sociohistorique et analyse textuelle. Denis HÜE (La traversée vers l'Angleterre dans l'“Estoire del saint Graal": déclinaisons d'un motif, pp. 514-529) analyse la fonction de la traversée vers l'Angleterre dans l'Estoire del saint Graal, en s'attardant en particulier sur la fonction des pièces de tissus (sein d'Abraham, chemise de Josephé). Le contexte et les modalités de l'arrivée de César dans 
la ville d'Abladane (Amiens) dans le roman homonyme et l'imaginaire projeté sur elle par la fiction sont au cœur de l'article de Colette VAN COOLPUT-STORMS (Le "Roman d'Abladane", passé-présent légendaire d'une ville du Nord, pp. 530-539). Le chapitre $46 \mathrm{du}$ livre I du Songe du Verger, organisé autour du mot un, est soumis à une analyse inspirée à la sémantique et à la psychomécanique du langage par Olivier SOUTET (Politique et grammaire: commentaire du paragraphe 46 du livre I du "Songe du Verger", pp. 540-551). Sylvie LEFÈVRe ("Jean de Saintré" et l'histoire, ou de la valeur d'un 'inssident', pp. 552-563) étudie l'épisode du combat de Saintré et de Boucicaut contre deux chevaliers italiens, décrit dans une courte digression historique à l'intérieur du ms. Paris, BnF, fr. 10057, en le comparant avec la version du même duel fournie par le Livre du Chevalier errant de Thomas III de Saluces. La dimension religieuse du Jouvencel dans ses retombées sur la conception de la noblesse et la chevalerie occupe Élisabeth PINTO-MATHIEU ("Unum corpus sumus in Christo": chevalerie et défense de la foi dans le "Jouvencel" de Jean de Bueil, pp.564-575). Élisabeth GAUCHER-RÉMOND analyse l'autoportrait ironique et plus généralement la parole antiphrastique à valeur cathartique chez Rutebeuf, Jean Régnier, Charles d'Orléans et Villon (Autobiographie et autodérision au Moyen Âge, pp.576-586). La question de la valeur de la poésie lyrique comme témoignage historique est au centre de la contribution de Clothilde DAUPHANT (Chanter en travaillant: Eustache Deschamps, bailli royal et vertueux poète, pp. 587-596); les deux derniers articles de cette section sont consacrés à François Villon (Jacqueline CERQUIGLINI-TOULET, Chien Cerberuz a quatre testes: François Villon et l'art de la discordance, pp. 597-609; Jean-Marie FRITZ, Des "Fioretti" aux "Lais": une poétique de l'ascèse et du dessaisissement, pp. 610-624).

5 La quatrième section renferme vingt études, consacrées en grande partie à des textes brefs; les deux premières contributions ont un caractère philologique: Géraldine VEYSSEYRE (Les "Merveilles de l'île de Bretagne", traduction partielle de l'"Historia Brittonum", pp. 627-640) fournit l'édition critique de la traduction de la septième partie de l'Historia Brittonum du Pseudo-Nennius d'après le ms. London, BL, Lansdowne 241, tandis qu'Hélène TÉTREL recherche la source de la petite digression consacrée à l'histoire de Bretagne dans l'Histoire ancienne jusqu'à César ( $Y$ a-t-il un «Roman de Brut» dans la première version de l'“Histoire ancienne jusqu'à César"?, pp. 641-649). Jean-Yves TILLETTE se focalise ensuite sur le sens et l'intention d'un dialogue latin en vers à caractère comique, réécriture d'un exemplum figurant dans la Disciplina clericalis de Pierre Alphonse (La revanche d'Abélard? Note sur le 'conte à rire' "De clericis et rustico", pp. 650-667) et JeanPierre BORDIER s'interroge sur les aspects performatifs de l'épisode de la bataille entre émirs sarrasins et chevaliers chrétiens figurant à la fin du premier mouvement du Jeu de saint Nicolas de Jean Bodel, qui aurait pu être joué dans les écoles cathédrales arrageoises (L'étrange bataille du "Jeu de saint Nicolas", pp. 668-677). La portée didactique d'un fabliau comique mettant en scène une histoire au caractère extraordinaire mais dépourvue de dimension merveilleuse est mise en évidence par Richard TRACHSLER ( $L e$ monde comme il va, ou du mauvais ménage du fabliau et de la merveille, pp.679-685). L'univers renardien est au centre des articles de Gérard GROS ( $L a$ fable de la branche X, "Renart et le vilain Liétart": un conte assumé par un prêtre, pp. 686-695), d'Astrid GUILLAUME (Reinhart Fuchs: sémiotique de la ruse, pp. 696-706) et de Bénédicte MILlAND-Bove (De Paulin à Paula: "Les aventures de Maître Renart et d'Ysengrin son compère" de Paulin Paris, pp. 707-716). Une étude consacrée au Bliocadran, court texte qui sert de prologue au Perceval de Chrétien de Troyes dans une partie de la tradition, et le déplacement de perspective qu'il suggère pour la lecture du roman (Nathalie KOBLE, Mireille sÉGUY, «Un 
nid pour quoi faire» à l'orée de "Perceval": les inventions de "Bliocadran", pp. 717-739) clôt la série d'études centrées sur les textes courts. Dans les pages suivantes, une analyse de la fonction des traductions de textes religieux pour la naissance, au XIII ${ }^{\mathrm{e}}$ siècle, des premiers romans en prose (Marie-Pascale HALARY, Ce que la traduction romane fait à la prose romanesque, pp. 740-751) est suivie de trois articles consacrés à l'interférence d'autres genres littéraires dans le développement de la matière arthurienne; Servane RAYNE-MICHEL montre l'importance du De doctrina christiana de saint Augustin pour l'interprétation des romans arthuriens (Gauvain l'analphabète, ou l'épreuve de la lecture selon saint Augustin, pp. 752-760); Christine FERLAMPIN-ACHER analyse l'interférence du savoir de type encyclopédique dans Artus de Bretagne, particulièrement pour ce qui est de la toponymie (Le Sorelois oriental d'“Artus de Bretagne", pp. 761-774), tandis que la naissance de la légende touchant la mère de Charlemagne, native de Hongrie, occupe Valérie FASSEUR (Berthe aux pieds hongrois. Révérences littéraires et forgerie de l'histoire au XIII siècle, pp. 775-778). Maria Luisa MENEGHETTI revient sur l'interprétation de quelques scènes des frises de la cathédrale de Fidenza pour proposer d'y voir une alternance entre figurations romanesques et moralisantes (Berte, Milon et Rolandin: entre écriture, image et moralité, pp. 789-800) et Joëlle Ducos analyse la diffusion du De re militari de Végèce à partir d'un nouveau recensement des témoins qui nous ont transmis la traduction de Jean de Meun (Jean de Meun au service de la culture des princes, pp. 801-817). Le Martyrologue des faulses langues composé par Guillaume Alecis à la fin du $\mathrm{xv}^{\mathrm{e}}$ siècle, qui serait apparenté au genre de la satire ménippée, et plus particulièrement le statut du narrateur et la représentation du rêve comme image de la quête de vérité font l'objet de l'article de Mireille DEMAULES (Une satire en forme de rêve: le "Martyrologue des faulses langues" de Guillaume Alecis, pp. 818-828). Les questions liées à la théâtralisation de l'historiographie antique dans cinq textes de la procession de Lille, abordées par Estelle DOUDET (Historier Rome: Valère Maxime au théâtre, pp. 829-838), et l'examen des qualifications de l'Inde dans la rédaction française du Devisement $d u$ monde mené par Danièle JAMES-RAOUL (Qualifier le monde dans le "Livre d'Ynde" de Marco Polo, pp. 839-854) achèvent la partie médiévale de ce riche recueil de mélanges. 